staffing, finance and so on, where ascertainable. Interesting lists of animals bred in zoos during 1965, and of zoological and veterinary research recently published or still in progress, also appear. A census of rare animals in the zoos of the world during 1966 is given so that a co-ordinated international breeding policy for rare animals could be drawn up, and inter-zoo matings or exchanges arranged. The criterion of rarity is the list of animals that are rare, or presumed to be rare, drawn up by the International Union for the Conservation of Nature. The recent attempt to breed from the giant pandas now in London and Moscow, which received such wide publicity, shows the action that the census aims at promoting. Stud books for eleven species of rare animals are now established-for some species, such as the wild horse and the wisent, zoos have reversed their usual role as consumers of rare animals and become producers.

The book ends with several appendices, including a dunce's corner in which is posted a list of the zoos that failed to answer questionnaires, or sent no information for the list of rarities. The volume is well produced, and is illustrated with excellent photographs, line drawings and diagrams. Misprints are few, and, although articles from so many contributors vary in quality, the standard required by the Yearbook Advisory Committee is well maintained.

L. Harrison matthews

\section{ADAPTIVE CONTROL}

\section{Theory of Self-Adaptive Control Systems}

Edited by P. H. Hammond. (Proceedings of the Second IFAC Symposium, September 14-17, 1965, National Physical Laboratory, Teddington, England.) Pp. xii + 363. (Pittsburgh: Instrument Society of America, 1966. Distributed by Plenum Press, New York.) \$15.

"ADAPTIVE" is a word with different meanings for different people. During the past decade its use has become widespread among control engineers and many papers about adaptive control systems have appeared in the engineering literature. The proceedings of the 1965 Symposium on the "Theory of Self-Adaptive Control Systems" organized by the control engineers' main international organization, the International Federation of Automatic Control (IFAC), contains a fair sample of the types of problem that control engineers have been describing as adaptive.

The original motivation for engineers to become interested in adaptive systems was the need to solve practical problems that were beyond the scope of classical design procedures. Examples of such problems are the design of safe control systems for very high performance military aircraft and the control, for maximum yield or maximum profit, of highly complex industrial chemical processes. These are problems where some piece of information that is required by the classical design procedure is missing, either because of prior uncertainty or because of unpredictable changes in the environment. The idea was that classical controllers might nevertheless be used if they ware made with variable parameters that could auto$m$ xtically be adjusted by a signal based on some measurement devised to compensate for the missing information. Various schemes of measurement and compensation have been proposed and two well-defined problems that have emerged are the problem of identifying the dynamics of an unknown system or "black box" and the problem of extremum control or "hill-climbing". These topics account for about half of the thirty-seven papers in the symposium proceedings.

When they were first proposed it seemed obvious that such systems, where a conventional controller is automatically adjusted so as to improve its own performance, should be classified as adaptive. It was soon pointed out, however, that the instrumentation for automatically adjusting the controller was merely an additional feed- back loop, and that systems described as adaptive and new in principle could equally well be described as multiloop nonlinear systems that were in principle no different from, although in practice more complex than, conventional feedback control systems. There was considerable controversy about whether the word adaptive could have any specific meaning for control engineers; one suggestion was that a control system was adaptive if its designer regarded it as being adaptive.

The interest of control engineers in adaptive systems was matched by a corresponding interest of applied mathematicians concerned with the mathematical theory of optimal control. The mathematical theory is not always directly applicable to practical engineering problems, but it does provide a basis for defining and classifying different types of control problem. In this context the name adaptive is reserved for the class of control problems where there is some prior uncertainty that can, with advantage, be reduced by observing the performance of the system as it operates: an adaptive system is thus a learning system. Only two papers at the symposium dealt with this well-defined class of adaptive control problems.

One of the attractions of the field of adaptive control systems is the possibility it offers for collaboration between control engineers and biologists. Engineers can offer techniques for analysing and synthesizing adaptive systems; biologists can describe adaptive systems that are more complex, more efficient and more reliable than any known to engineering. There is a section in the proceedings of five papers on adaptive control in biological systems.

Other papers in the proceedings deal with problems that are related to adaptive control problems. It is clear that there is much research in progress under the title of "Adaptive Control" and that this covers a wide range of related problems. These proceedings show that the IFAC symposium was successful in bringing together a variety of these problems and that there is a continuing need for good understanding of the relationships between them

O. L. R. JACOBS

\section{PROGRESS OF GAS DYNAMICS}

\section{Rarefied Gas Dynamics}

Edited by C. L. Brundin. (Proceedings of the Fifth International Symposium held at the University of Oxford, 1966. Advances in Applied Mechanics Supplement 4.) Vol. 1. Pp. $x x+879$. \$18.50. Vol. 2. Pp $\mathrm{xx}+881-1731$. 148s. (New York: Academic Press, Inc.; London: Academic Press, Inc. (London), Ltd., 1967.)

THese two volumes are made up of the written versions of 103 papers read at the Fifth International Symposium on Rarefied Gas Dynamics; all the papers which were read are represented.

The range of topics covered by such a large number of papers must clearly be very wide, so that the two volumes are organized into five sections, four of which commence with a review paper, written by an eminent contributor to the specialized field in question, and designed to give perspective to the research papers which follow. Divisions between sections (and sub-sections) are not rigid and several papers may as well be in one place as another. This fact is representative of the thread of unity which runs through this very interesting and flourishing branch of modern gas dynamics.

The first section, introduced by F. C. Hurlbut, is devoted to an examination of gas-surface interactions. The subject has long been studied from the experimental point of view, especially to obtain information on energy and momentum accommodation coefficients. The need for a deeper understanding of the complexities of the interaction represented by these coefficients has lately led to the development of theoretical models. Both 